\section{Case Reports in Ophthalmology}

\title{
Unusual Case of Vogt-Koyanagi- Harada Disease Associated with SAPHO Syndrome: A Case Report
}

\author{
Miyuki Hasegawa Akira Fukutomi Akiko Jinno Kyoko Fujita \\ Motohiro Kamei \\ Aichi Medical University, Department of Ophthalmology, Nagakute City, Japan
}

\section{Keywords}

Vogt-Koyanagi-Harada disease - SAPHO syndrome - Choroidal detachment - Serous retinal detachment . Cyclosporine

\begin{abstract}
A 66-year-old Japanese woman who was diagnosed with synovitis-acne-pustulosis-hyperostosis-osteitis (SAPHO) syndrome presented with bilateral blurred vision 4 months prior to visiting our hospital. She had visited a local ophthalmology clinic first. She was diagnosed with conjunctivitis and was prescribed antibacterial eye drops. The symptoms persisted in spite of treatment. She was then referred to our hospital. At her initial visit, the visual acuities were 0.6 in both eyes. A slit-lamp examination revealed bilateral shallow anterior chamber, and intraocular pressures of $18 \mathrm{~mm} \mathrm{Hg}$ in the right eye and $16 \mathrm{~mm} \mathrm{Hg}$ in the left eye. There were no cells in the anterior chamber. Fundus examination revealed bilateral annular choroidal detachment and serous retinal detachment. Fluorescein angiography showed leakage of dye from the retinal pigment epithelium (RPE) and indocyanine green angiography showed focal choroidal hypoperfusion. Optical coherence tomography showed wavy RPE line and blurry thick choroid. Systemic investigation by the physician demonstrated bilateral pleural effusions of unknown origin. The patient had a past history of breast cancer; however, no metastasis was identified via malignant cells through cytology, laboratory findings, radiographs, CT, and MRI. After the diagnosis of Vogt-Koyanagi-Harada (VKH) disease was made, the patient was treated with local and systemic steroid including high-dose intravenous corticosteroids, and $150 \mathrm{mg}$ of cyclosporine per day. Seventy days after the second high-dose of intravenous corticosteroids, these medications brought a complete resolution of both choroidal and reti-
\end{abstract}




\section{Case Reports in Ophthalmology}

nal detachment. VKH disease associated with SAPHO syndrome is rare. The combination of immunosuppressive drug and steroid might be helpful for severe cases of VKH disease.

\section{Introduction}

Synovitis-acne-pustulosis-hyperostosis-osteitis (SAPHO) syndrome is a rare but clinically important disease characterized by pustular skin lesions and non-pyogenic osteoarticular lesions [1]. The etiology of SAPHO syndrome remains unclear, but neutrophil activation is suggested to contribute to the pathophysiology of this disease [2]. Ocular inflammation associated with SAPHO syndrome is rare. In this report, we describe an unusual case of VogtKoyanagi-Harada (VKH) disease manifesting bilateral annular choroidal detachment and exudative retinal detachment in a patient with SAPHO syndrome.

\section{Case Report}

A 66-year-old Japanese woman presented with bilateral blurred vision 4 months prior to visiting our hospital. She had been diagnosed with conjunctivitis and prescribed antibacterial eye drops by a local ophthalmology clinic. While undergoing therapy, she developed general fatigue and lumbar pain. She visited a private clinic, and was diagnosed with bilateral exudative pleural effusions. She was then referred to our hospital for further investigation and treatment. After admission to our hospital, complete physical examinations were done. Her exudative pleural effusion was of unknown origin. The patient had a past history of breast cancer 2 years ago, but no malignant cells were observed in cytology examination, and no malignancy was detected by CT and MRI. In pleural fluid examination, the specific gravity was 1.304 , cell count $397 / \mu \mathrm{L}$ (neutrophils $4.0 \%$, lymphocyte $62.0 \%$, macrophage $34.0 \%$ ), lactate dehydrogenase $105 \mathrm{U} / \mathrm{L}$, adenosine deaminase $8.7 \mathrm{U} / \mathrm{L}$, total protein $4.4 \mathrm{~g} /$ $\mathrm{dL}$, rheumatoid factor $<10.0 \mathrm{IU} / \mathrm{mL}$, antinuclear antibodies $( \pm)$, and negative cultures. The cause of lumbar pain was also examined by the orthopedic department, and she was diagnosed with multiple spondylitis. Findings of an MRI of the spine and bone scintigraphy are shown in Figure 1. She also had palmoplantar pustulosis beginning several years ago. Based on the findings of spondylitis and palmoplantar pustulosis, she was diagnosed with SAPHO syndrome by both orthopedists and dermatologists, and was prescribed antibiotics and nonsteroidal anti-inflammatory drugs (NSAIDs). Seven days after admission, she was referred to ophthalmology for investigation of the cause of her blurred vision. At presentation, her bestcorrected visual acuity (BCVA) was 0.6 in both eyes. Intraocular pressure was $18 \mathrm{~mm} \mathrm{Hg}$ in the right eye and $16 \mathrm{~mm} \mathrm{Hg}$ in the left eye. Slit-lamp examination demonstrated bilateral shallow anterior chambers. There were no keratic precipitates in both corneas, and no cells in the anterior chambers. Fundus examination revealed bilateral annular choroidal detachment with exudative serous retinal detachment involving the macula. Optical coherence tomography (OCT) showed subretinal fluid with a wavy retinal pigment epithelium (RPE) line and thicker choroid in both eyes. Fluorescein angiography (FA) depicted multiple areas of pinpoint dye leakage from the RPE. Indocyanine green angiography (ICGA) showed multiple areas of choroidal hypoperfusion in both eyes (Fig. 2). B-scan ultrasonography confirmed choroidal detachment with no associated scleral thickening or masses affecting the choroid and sclera. Although the patient did not meet all the diagnostic criteria for VKH disease, dif- 


\section{Case Reports in Ophthalmology}

Case Rep Ophthalmol 2018;9:202-208

DOI: $10.1159 / 000487226$

ferential diagnosis showed no evidence of diseases such as uveal effusion syndrome, posterior scleritis, or choroidal metastasis, and laboratory markers of other types of uveitis including sarcoidosis, syphilis, and tuberculosis were negative. Moreover, autoimmune diseases such as rheumatoid arthritis and antineutrophil cytoplasmic antibody-associated vasculitis, which are often accompanied by uveitis, were also unlikely because of the absence of antibodies specific to these diseases. Her clinical findings including FA, ICGA, and OCT indicated VKH disease. Treatment was begun with a subtenon injection of triamcinolone acetonide in both eyes, while internists continued investigations to rule out other diseases. After 7 days, when internists finished the diagnostic workup with no additional findings, highdose intravenous corticosteroids were given for 3 days. Treatment was continued with a slowly tapering course of oral prednisolone to $30 \mathrm{mg}$ per day for 8 days. Because the choroidal and retinal detachments persisted in spite of steroid therapy, the patient was prescribed immunosuppressive medication of cyclosporine $150 \mathrm{mg}$ twice daily. Two weeks after starting cyclosporine, cellulitis occurred in the left leg and C-reactive protein (CRP) increased to $23.5 \mathrm{mg} / \mathrm{dL}$. Cyclosporine was discontinued, and prednisolone was further tapered to $10 \mathrm{mg}$ per day. Because of this episode, ocular findings persisted and worsened. Ten days after discontinuing cyclosporine, her general condition improved and CRP level decreased to within the normal limit. Treatment was resumed with high dose $(1 \mathrm{~g})$ of intravenous corticosteroids for 3 days and cyclosporine $150 \mathrm{mg}$ twice daily. Seventy days after the second course of high-dose intravenous corticosteroids, choroidal and retinal detachments were resolved completely, and BCVA was 0.5 in the right eye and 0.7 in the left eye (Fig. 3). Bilateral pleural effusions were also resorbed, and her general condition improved.

\section{Discussion}

First described by Chamot and associates [1] in 1987, SAPHO syndrome is a disease characterized by pustular skin lesions and nonpyogenic osteoarticular lesions. At present, SAPHO syndrome is regarded as a rare disease with an estimated prevalence of less than 1 in 10,000 [2]. Furthermore, ocular inflammation associated with SAPHO syndrome is rare. Fernández-Melón and associates [3] reviewed 394 patients with uveitis having spondyloarthropathies and reported only one patient with SAPHO syndrome. Yabe and associates [4] reported two cases of SAPHO syndrome accompanied by Behçet's disease. Tanaka and associates [5] reported a case of scleritis associated with SAPHO syndrome. There are no reports of VKH disease with exudative choroidal and retinal detachments associated with SAPHO syndrome. In this report, we describe an unusual case of VKH disease-associated SAPHO syndrome.

The ocular findings in our case, such as annular choroidal detachment, were uncommon compared to typical VKH disease. There are a few reports of VKH disease with annular choroidal detachment [6, 7]. Yamamoto and associates [6] reported a case of annular choroidal detachment without retinal detachment associated with VKH disease. Elaraoud and associates [7] reported a similar case. Our case did not manifest overt retinal detachment or disc swelling but showed multiple areas of fluorescein leakage from the RPE on FA and choroidal hypoperfusion areas on ICGA. OCT revealed increased choroidal thickness and a wavy RPE line. Ultimately, we diagnosed the patient with VKH disease associated with SAPHO syndrome.

Although several factors have been speculated to be possible etiologies of the SAPHO syndrome, such as infection, genetic predisposition, and immunological dysfunction, they 
remain controversial. The relationship between SAPHO syndrome and VKH disease is also unclear. Several studies have demonstrated that HLA-DR4 is strongly associated with VKH disease $[8,9]$. On the other hand, there is no report of HLA-DR4-positive individuals with SAPHO syndrome. However, the association of HLA types with diseases remains controversial, because there are many questions regarding HLA typing for those diseases. VKH disease is a granulomatous inflammation related to $\mathrm{T}$ cell-mediated autoimmune disease targeting melanocyte self-antigens [10]. Grosjean and associates [11] have reported that autoantibodies were found in 20 patients $(22.2 \%)$ with SAPHO syndrome, and they mentioned a link between autoimmunity and SAPHO syndrome. The mechanism of overlapping multiple autoimmune disorders is unknown. Infections or stress factors presumably activate humoral immunity, and the development of multiorgan immune disease is a possibility.

Our case did not respond to steroids alone, and immunosuppression therapy with cyclosporine was considered. NSAID is regarded as the first-line treatment for SAPHO syndrome [12]. For patients who respond insufficiently to NSAIDs, addition of corticosteroids, bisphosphonates, and disease-modifying anti-rheumatic drugs such as methotrexate, sulfasalazine, and cyclosporine can be considered [12,13]. Moreover, biologics such as tumor necrosis factor inhibitors may be used in the case of refractory disease [12, 14]. In our case, steroid therapy was suspended due to complication with cellulitis during treatment. We must always keep in mind systemic conditions and the adverse effects of steroids. In our case, although ophthalmic findings and general conditions did not improve with NSAIDs and steroids, both were improved by combination with cyclosporine.

In conclusion, we reported a rare case of VKH disease associated with SAPHO syndrome. Combined therapy of immunosuppressive drugs with steroids was effective in resolving choroidal and retinal detachments.

\section{References}

1 Chamot AM, Benhamou CL, Kahn MF, Beraneck L, Kaplan G, Prost A: Acne-pustulosis-hyperostosisosteitis syndrome. Results of a national survey. 85 cases. Rev Rhum Mal Osteoartic 1987;54:187-196.

2 Magrey M, Khan MA: New insights into synovitis, acne, pustulosis, hyperostosis, and osteitis (SAPHO) syndrome. Curr Rheumatol Rep 2009;11:329-333.

3 Fernández-Melón J, Muñoz-Fernández S, Hidalgo V, Bonilla-Hernán G, Schlincker A, Fonseca A, Vieitez J, Martín-Mola E: Uveitis as the initial clinical manifestation in patients with spondyloarthropathies. J Rheumatol 2004;31:524-527.

4 Yabe H, Takano Y, Nomura E, Nakayama M, Kihara M, Miyakawa S, Horiuchi Y: Two cases of SAPHO syndrome accompanied by classic features of Behcet's disease and review of the literature. Clin Rheumatol 2008;27:133-135.

5 Tanaka R, Sakurai K, Kaburaki T: Scleritis associated with SAPHO syndrome: a case report. Ocul Immunol Inflamm 2017;6:1-3.

6 Yamamoto N, Naito K: Annular choroidal detachment in a patient with Vogt-Koyanagi-Harada disease. Graefes Arch Clin Exp Ophthalmol 2004;242:355-358.

7 Elaraoud I, Andreatta W, Jiang L, Damer K, Al-Ibrahim J: A mystery of bilateral annular choroidal and exudative retinal detachment with no systemic involvement: is it part of Vogt-Koyanagi-Harada disease spectrum or a new entity? Case Rep Ophthalmol 2017;16:1-6.

8 Shindo Y, Inoko H, Yamamoto T, Ohno S. HLA-DRB1 typing of Vogt-Koyanagi-Harada's disease by PCRRFLP and the strong association with DRB1*0405 and DRB1*0410. Br J Ophthalmol 1994;78:223-226.

9 Shi T, Lv W, Zhang L, Chen J, Chen H. Association of HLA-DR4/HLA-DRB1*04 with Vogt-KoyanagiHarada disease: a systematic review and meta-analysis. Sci Rep 2014;4:6887.

10 Moorthy RS, Inomata H, Rao NA. Vogt-Koyanagi-Harada syndrome. Surv Ophthalmol 1995;39:265-292.

11 Grosjean C, Hurtado-Nedelec M, Nicaise-Roland P, Ferreyra-Dillon R, Bollet C, Quintin E, Dieude P, Palazzo E, Wattiaux MJ, Kahn MF, Meyer O, Chollet-Martin S, Hayem G. Prevalence of autoantibodies in SAPHO syndrome: a single-center study of 90 patients. J Rheumatol $2010 ; 37: 639-643$. 
Yabe H, Ohshima H, Takano Y, Koyanagi T, Usui H, Nojiri K, Ochi K, Kihara M, Horiuchi Y: Mucosal lesions may be a complication of SAPHO syndrome: a study of 11 Japanese patients with SAPHO syndrome. Rheumatol Int 2010;30:1277-1283.

14 Moll C, Hernández MV, Cañete JD, Gómez-Puerta JA, Soriano A, Collado A, Sanmartí R: Ilium osteitis as the main manifestation of the SAPHO syndrome: response to infliximab therapy and review of the literature. Semin Arthritis Rheum 2008;37:299-306.
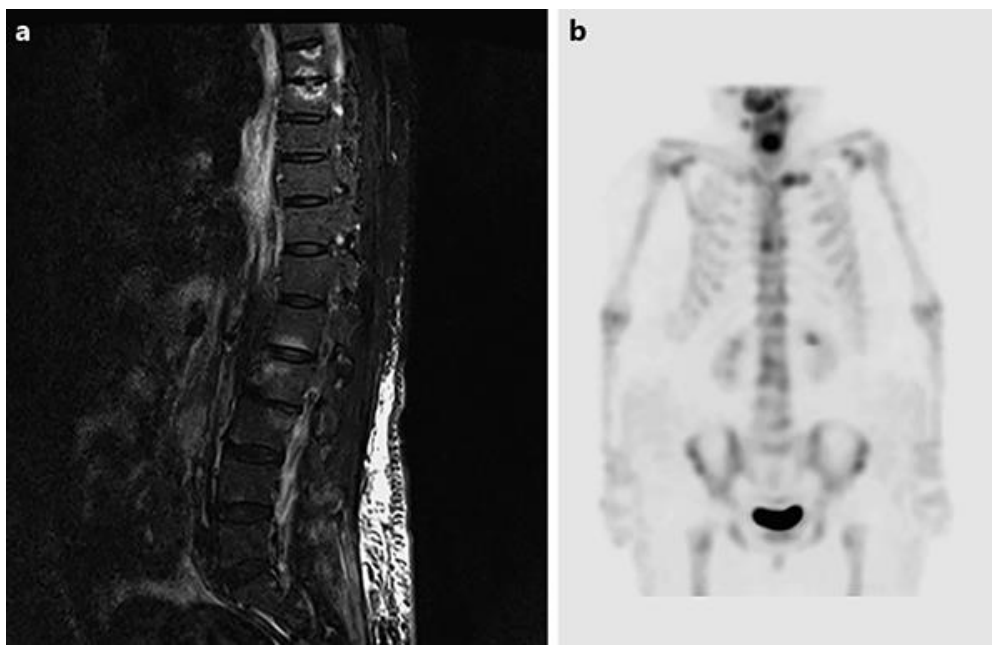

Fig. 1. a MRI T2-weighted image of the spine shows hyperintensity in Th7, Th8, L2, and L3. b Bone scintigraphy shows abnormal uptake in sternoclavicular joint, C7, Th1, Th7, Th8, Th12, and L3. 


\section{Case Reports in Ophthalmology}

\begin{tabular}{l|l}
\hline Case Rep Ophthalmol 2018;9:202-208 \\
\hline DOI: 10.1159/000487226 & $\begin{array}{l}\text { ○ 2018 The Author(s). Published by S. Karger AG, Basel } \\
\text { www.karger.com/cop }\end{array}$ \\
\hline
\end{tabular}

Hasegawa et al.: Vogt-Koyanagi-Harada Disease Associated with SAPHO Syndrome
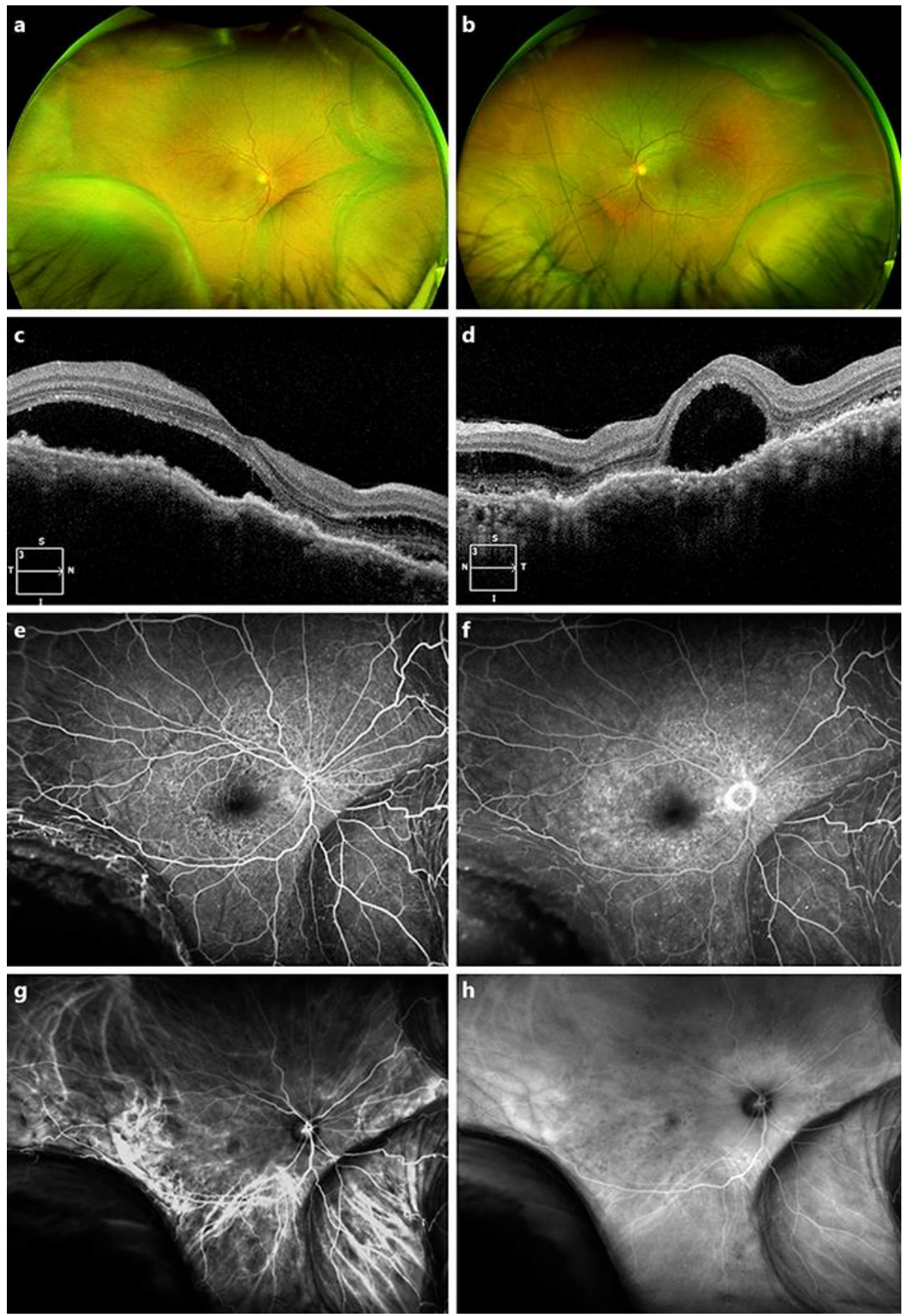

Fig. 2. Color fundus photographs (a, b) and optical coherence tomography images (c, d) at initial visit. Fundus photographs show annular choroidal detachment and serous retinal detachment in both eyes. Optical coherence tomography images show a wavy retinal pigment epithelium and thick choroid in both eyes. Fluorescein angiography and indocyanine green angiography findings. Early-phase fluorescein angiogram of right eye (e) shows dye leakage from retinal pigment epithelium. Late-phase fluorescein angiogram (f) shows slightly increased dye leakage. Dye pooling is not seen. Early- and late-phase indocyanine green angiograms ( $\mathbf{g}$ and $\mathbf{h}$, respectively) of right eye show focal hypoperfusion areas. Similar changes were seen in the left eye. 


\section{Case Reports in Ophthalmology}
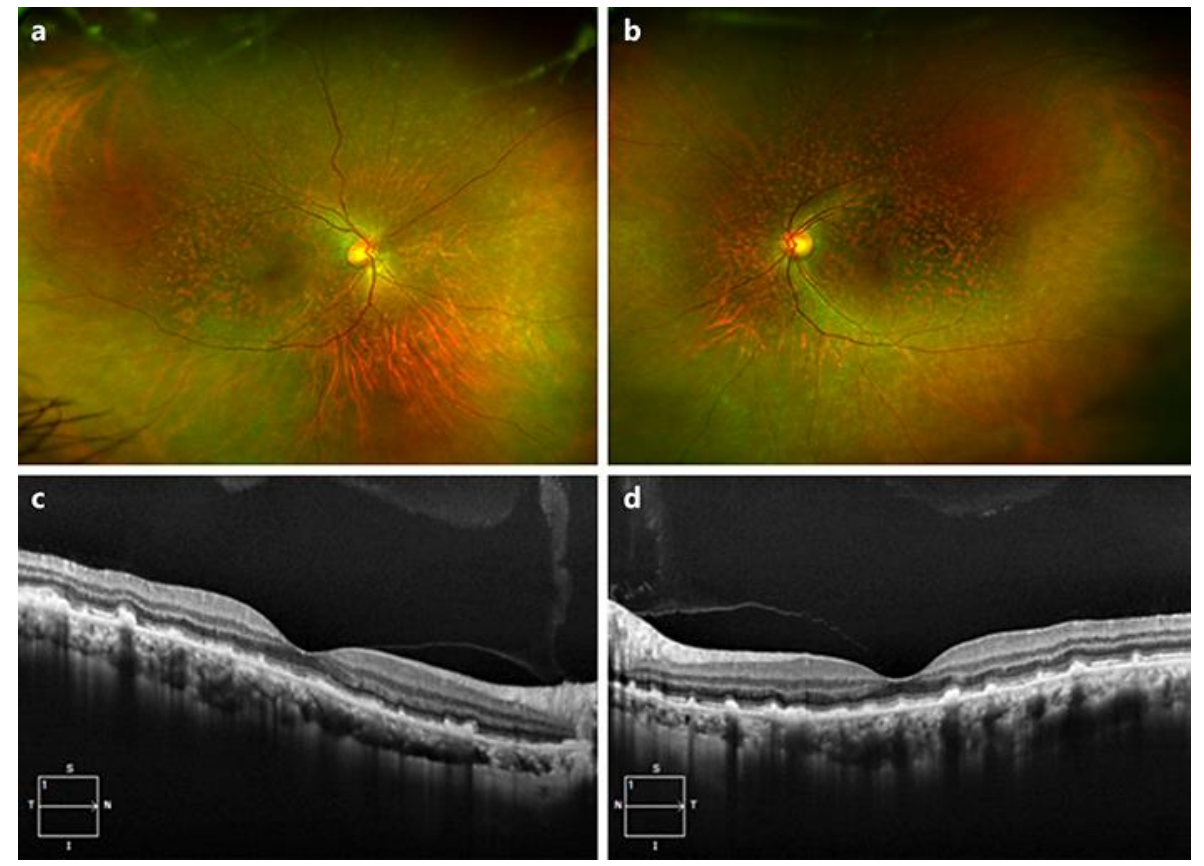

Fig. 3. Color fundus photographs (a, b) and optical coherence tomography images (c, d) at 70 days after initial treatment. Fundus color photographs show resolved annular choroidal detachment and serous retinal detachment in both eyes. Optical coherence tomography images show resolved serous retinal detachment. 